June 19th. Carbohydrate 15, protein 38, fat 28-calories 464. Sugar in the afternoon.

June 20th. Insulin, 20 minims. Carbohydrate 14, protein 54, fat 45-calories 677. No sugar in afternoon.

June 21st. Some sugar in the morning. Insulin, 22 minims, given. Carbohydrate 24 , protein 60 , fat 106-calories 1,290 . No sugar rest of the day.

June 22nd. A little sugar after breakfast. Insulin, 22 minims, given. Carbohydrate 26 , protein 60 , fat 130 -calories 1,514 . No sugar rest of the day.

June 23rd. Sugar present in the morning. Insulin, 23 minims, giren. Carbohydrate 26 , protein 60 , fat 140 -calories 1,604 . No sugar rest of the day.

June 24th. Very slight sugar in the morning. Insulin, 23 minims, given. Carbohydrate 30 , protein 64 , fat 132 -calories 1,504. Sugar absent rest of the day.

June 25th. Sugar at 9 and 11 a.m. Insulin, 30 minims, given. Dict as on June 24th. No sugar rest of the day.

June 26th. Trace of sugar at 11 a.m. Insulin, 30 minims, giren. Carbohydrate 25, protein 75 , fat 161 -calories 1,850 . No sugar later.

That was the last date on which the injection was given in the patient's bedroom, for as he was feeling extremely well and anxious to be again at business I suggested that he should come to my house at 5 p.m., the time when in the ordinary course he would be home for his evening meal, receive his injection then, and go home at once, where he would arrive within the stipulated period of fifteen minutes, which is the maximum that can elapse without food being taken after a dose has been administered. This he did for a week with the same dose, and a diet value of 2,000 calories; I then left him for a week-end to administer his own injection, which he did for two days with such success that he now undertakes his own case, and has improved on the plan by adopting my suggestion of giving a morning and evening dose, which he assures me is better, in that he feels fresh all day, whereas formerly he felt much less vigorous in the forenoon; it will also mean a reduction in expense which he estimates at $£ 15$ a year, because he can save in dosage at least 4 minims a day.

Only once has he felt any ill effects, and this was when in his enthusiasm he took a 20 -minim dose in the evening after a similar forenoon dose, and in three hours after had a horrible feeling of "illness" with buzzing in the ears and profuse cold sweat-obviously overdosage; he was promptly relieved by drinking a tablespoonful of sugar and water. He has learned from this that a trace of sugar in the morning urine is safe, and that he must never hope for, nor try to get, a constantly sugar-free urine.

$\mathrm{He}$ has now been at business for ten weeks and feels it no strain, being able even to return to the office for two hours in the evening twice a week. He looks well, walks with a sprightly step, and his weight is 9 st. $10 \frac{1}{2} \mathrm{lb}$.

\section{Practical Conclusions.}

1. Two days' fasting, and then a "ladder diet" until the patient's urine begins to show sugar, gives the starting point for the first insulin injection, which $I$, in future, will give twice a day, starting with 20 minims in the day.

2. Food must be ready for the patient as soon as he receives his injection, and in no circumstances can longer than fifteen minutes be allowed to elapse.

3. The patient must always have sugar and water at his bedside, and be advised to take a small amount should symptoms of faintness, depression, air hunger, etc., appear.

4. He must be educated to the testing of his urine for sugar and keep a record of every specimen voided for the first two weeks and morning and evening thereafter.

5. Do not start if there is any fear that the treatment cannot be kept up, and it can never be forgotten that at present the cost of the insulin will entail an expenditure of at least $£ 90$ per annum.

6. Do not attempt to get a constantly sugar-free urine. A trace of sugar in the morning specimen is a safeguard giving a feeling of assurance that no harm can result from the treatment.

7. It cannot be forgotten at any time that the treatment of diabetes is still a matter of careful dieting, and that insulin is only our greatest and most valuable aid.

\section{A TWO-LOOP SUTURE.}

JOHN CRAIG, F.R.C.S.I.,

CONSULTING SURGEON, GISBORNE HOSPITAL, NEW ZEALAND.

Is the Jourval of June 9th, 1923 (p. 969), is publislied an article by Mr. W. G. Spencer describing a two-loop button suture. Below I give a description of a stitch which I invented and published in the New Zealand Medical Journal of August, 1914. It appears to me that Mr. Spencer's twoloop suture is but an abridged form of mine. My suture seems capable of more extended use, especially as one hears reports from time to time of catgut having caused fatalities through tetanus or other infections. This method of mine could replace the use of catgut for closing abdominal wounds.

The first needle is inserted at the inner extremity of the line marked $\mathbf{A}$, and is passed between peritoneum (deep to the muscle) and fascia and brought out on the skin surface one-third of an inch from the skin margin. The second needle is passed directly through the peritoneum at the point of the line marked A, two continuous sutures being taken slightly sloping up, the last stitch passing through peritoneum, from within, and coming out at the point marked $\mathrm{C}$. It is then passed deep between peritoneum and muscle, coming to the surface one-third of an inch from the skin margin on the left side, as did the first needle. The two ends of the suture are drawn tight, bringing the peritoneal edges together. A clip forceps is applied to the ends of the ligature to keep it taut. The remaining sutures are placed in the same fashion, and about three of them will close most wounds. When the line of peritoneum is

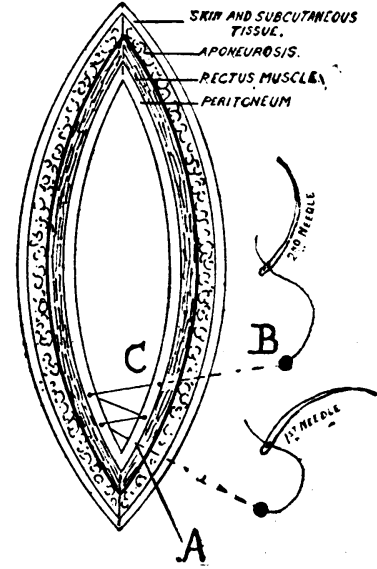

Dingram 1.

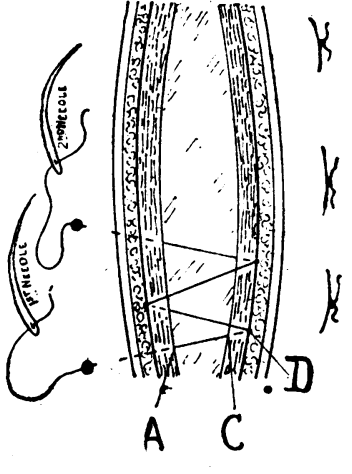

Diagrim 2. closed, the same process is repeated on the right side. In this case the first needle is passed under peritoneum and deep to muscle and fascia at the inner end of the line marked $\mathbf{A}$ in Diagram 2, and brought out one-third of an inch from the skin margin. The second needle is passed under the fascia, deep to muscle, at the point marked $\mathrm{C}$, then through to the surface of the aponeurosis. In all two continuous stitches are made, as shown in the diagrams. The ends of the suture are drawn tight and clipped until all are placed, when they are tied over a small pad of gauze or rubber tube cut short. When drawn tight the suture makes a short straight line easily removed by cutting one end of the loop close to the skin and pulling on the part that is left. The peritoneal suture may be removed in three days, the muscle suture in thirteen days or longer if deemed advisable.

The suture material may be silk, thread, or silkworm gut, all of which can be relied upon for asepsis as they stand plenty of boiling. The two-needle method is also a precaution to prevent any chance of infection from sebaceous or other ducts. If the same two needles are used for the next stitch they can be rapidly sterilized with pure carbolic acid or lysol and rinsed in hot water.

I have had better results with this method than with any other. Diagram 1 shows the peritoneal stitches being placed, Diagram 2 shows the peritoneum closed with the line of stitches and also shows the placing of the aponeurotic suture. If in an emergency there is cause for haste, the peritoneum may be included in the muscle stitch, thus having loops down one side of the wound only. One advantage of the separate peritoneal stitches is that their early removal prevents any irritation which might arise from the sutures impinging on the abdominal contents when there is only one layer that has to be retained for a considerable period. Round needles must be used, and they require to be of large size in fat subjects. The needles should always be passed from within out to secure asepsis. 